\title{
Lessons learned from inadequate implementation planning of team-based chronic disease management: implementation evaluation
}

\author{
Shannon L. Sibbald ${ }^{1,2^{*}}$, Rachelle Van Asseldonk ${ }^{1}$, Peiwen L. Cao ${ }^{1}$ and Benson Law ${ }^{1}$
}

\begin{abstract}
Background: This study was a retrospective evaluation of an unsuccessfully implemented team-based, chronic disease management program, with an aim to understand more about implementation barriers. The program, the Chronic Disease Management Initiative (CDMI) was a new collaborative model of care for patients with COPD. It utilized customized health information and interactive tools, mainly smartphones, for ongoing disease management. The program's goal was to demonstrate that integrated team-based models of care could improve patient care, as well as reduce readmission rates and emergency department visits. The program planning for CDMI began in 2017, followed by the implementation and evaluation period in 2018. After a 10-month implementation period, the program was unable to enroll a sufficient number of patients to examine if there was an improvement in patient outcomes.
\end{abstract}

Methods: A retrospective case-study design using multiple data sources was used to gather feedback from participants involved in CDMI. Data collection occurred throughout planning and implementation and continued into early 2019. Semi-structured interviews were conducted, and transcripts were analyzed using NVivo 10 software. This was followed by content analysis.

Results: Analysis revealed four key themes as barriers to CDMl's implementation: 1) lack of a needs assessment with key stakeholders; 2) lack of buy-in from medical staff; 3) inadequate patient engagement and; 4) contextual barriers. Planners did not conduct a proper needs assessment, nor include patients in the study design. In addition, there was insufficient consideration for how CDMI should be integrated into the usual COPD care plan, leading to confusion in roles and responsibilities. Poor communication between the implementation team and healthcare providers implementing the program, led to a lack of buy-in and engagement.

(Continued on next page)

\footnotetext{
* Correspondence: ssibbald@uwo.ca

${ }^{1}$ Faculty of Health Sciences, University of Western Ontario, London, Canada ${ }^{2}$ Department of Family Medicine, Schulich School of Medicine and Dentistry, University of Western Ontario, London, Canada
}

(c) The Author(s). 2021 Open Access This article is licensed under a Creative Commons Attribution 4.0 International License, which permits use, sharing, adaptation, distribution and reproduction in any medium or format, as long as you give appropriate credit to the original author(s) and the source, provide a link to the Creative Commons licence, and indicate if changes were made. The images or other third party material in this article are included in the article's Creative Commons licence, unless indicated otherwise in a credit line to the material. If material is not included in the article's Creative Commons licence and your intended use is not permitted by statutory regulation or exceeds the permitted use, you will need to obtain permission directly from the copyright holder. To view a copy of this licence, visit http://creativecommons.org/licenses/by/4.0/ The Creative Commons Public Domain Dedication waiver (http://creativecommons.org/publicdomain/zero/1.0/) applies to the data made available in this article, unless otherwise stated in a credit line to the data. 


\begin{abstract}
(Continued from previous page)
Conclusion: The key themes resonate with what is already known in the literature. This study supports the importance of using a theoretically grounded plan for implementation. Using a model only in the planning stages is insufficient even when an intervention is based on evidence to support higher quality care. It is imperative to consider practical and contextual factors of program implementation and their interactions. By detailing the 'failed implementation' of this intervention, we hope to share important lessons about the need to plan implementation processes early in program planning.
\end{abstract}

Keywords: Implementation study, COPD, Chronic disease, Change management, Evidence-based practice, Facilitation, Implementation research into practice, Failure

\section{Background}

The literature has demonstrated many complex and interconnected factors that can determine implementation success. These include supportive leadership, an enabling organizational culture, patients' perceptions on the intervention, amongst others [1-3]. It is also acknowledged that these factors interact with one another, although little is known about exactly how context might shift the balance of interconnectivity. The Consolidated Framework for Implementation Research (CFIR) specifically identifies the implementation setting as an important factor, alongside the interplay between individual stakeholders and facilitators [4]. However, more guidance on this interplay is needed. Implementation science is focused on improving the successful uptake of evidence-based methodologies to improve health care quality and overall effectiveness [5]. Evaluating unsuccessful implementations can provide lessons that can be applied prospectively to improve the likelihood of intervention success [6]. However, details of unsuccessful implementations are often under-reported, leaving notable gaps in the literature [7], negatively impacting the quality of care that patients receive, as well as contributing to increased costs and provider workload [8]. Motivated by this gap, we conducted a retrospective evaluation on an unsuccessfully implemented chronic disease management program.

The Chronic Disease Management Initiative (CDMI) program was guided by a chronic disease management model proposed by Bodenheimer and colleagues [9] with six elements: linkage with community resources, buy-in by health care organizations, structured practice teams for chronic care management, self-management support, decision support and clinical information systems that ensured reminders and feedback about patient progress.

CDMI was designed to support patients living with chronic obstructive pulmonary disease (COPD) through an interactive, mobile-based platform delivered in a primary care setting. Participants were given a smartphone for receiving targeted messages from their healthcare providers. As part of the program, patients and providers received a brief training on the use of the device during regularly scheduled visits. Text messages were initiated by a program navigator at least twice a week to reinforce health teaching and monitoring activities. Previous research has shown that some patients prefer text message communication to in-person or phone conversations because it can diminish feelings of embarrassment related to health issues [10]. Another study identified that being able to contact health care providers with a mobile device was like having a "permanently outstretched hand", even when health care services are not being accessed [11].

After a 10-month implementation period, the CDMI program was unable to enroll a sufficient number of patients to realize any improvement in patient outcomes. As such, researchers were unable to determine whether the smart phone technology, if implemented properly, could actually work to improve communication between patient and provider and, in turn, improve patient outcomes.

The contributions of this study to the implementation science literature include: 1) providing experiential knowledge to guide future health interventions in planning for real world challenges they may encounter, especially on the individual or organizational level; 2) highlighting the importance of involving participants from the outset of planning as it can improve the likelihood of a successful implementation and guide the intervention to fruition; and 3) bridging the gap in the literature where unsuccessful implementations are underreported.

\section{Methods}

Our research was guided by the Consolidated Framework for Implementation Research (CFIR) which helped to identify critical constructs in the implementation process [12]. CDMI's implementation team $(n=11)$ was made up of researchers $(n=2)$, family physicians $(n=2)$, a respirologist $(n=1)$, nurses $(n=2)$, psychiatrists $(n=$ $2)$, a cardiologist $(n=1)$, and a research coordinator $(n=1)$. 
The 10-month intervention period was led by the research coordinator and executed by a subset of the research team. After the unsuccessful implementation, all eleven team members were invited to take part in a semi-structured interview. Four informants participated in-person (36.36\% response rate), one participated via email; one explicitly chose not to partake since they believed that they were not integral to CDMI's implementation; the others absent did not cite a reason for their lack of participation.

Participants were asked eight open-ended questions about their view on the project's development and implementation, their involvement, team perceptions, as well as areas of improvement (See Additional file 1). Questions were developed to explore the constructs in CFIR [12]. The interviews were transcribed verbatim; NVivo10 was used to support analysis.

The interview data was coded by two researchers (SLS, PC) using a conventional content analysis [13]. The codes and themes were reviewed and refined by the entire research team. Analysis began with an initial readthrough of the transcripts to identify significant and relevant content. This process was repeated until the transcripts were fully coded and all relevant content was marked. Data analysis was augmented by looking at related implementation documents such as meeting minutes, project proposals, and ethics documents. Codes were combined into themes and meaningful patterns in the data were examined in relation to all data sources [14]. The STARi checklist by Pinnock and colleagues [15] was used to ensure transparent research reporting (See Additional file 2).

\section{Results}

Four themes were identified from the responses as key barriers to CDMI's implementation: 1) lack of needs assessments and engagement with key stakeholders; 2) lack of buy-in from medical staff; 3) inadequate patient engagement; and 4) contextual barriers. These themes were organized based on the progression of the implementation process.

\section{Lack of a needs assessment and engagement with key stakeholders}

All participants thought that a needs assessment with practitioners and patients, described as a crucial element of healthcare implementation, was absent from CDMI. Participants also believed that some key players of project implementation were not sufficiently engaged prior to implementation, such as the nurses who were ultimately responsible for delivering the technology-based intervention. Participants suggested that performing a needs assessment, prior to the implementation would have facilitated staff buy-in through the identification of possible challenges and barriers. This would also increase awareness of possible facilitators.

"...there being a needs assessment prior to the planning of the whole research, those needs could have been addressed and there would have been greater buy-in by the clinics... contacting the clinics saying that we would like to sit with them and create first of all, some sort of a questionnaire regarding needs assessment in order to assess the needs involved..." Participant 1.

Beyond a formal needs assessment, participants simply acknowledged that the opportunity for stakeholders to provide feedback and be heard was largely missing.

"We have to step back and make sure we've got the pathways and models of care really, fully engaged... making sure that we engage the key players in each of the family health teams. I think we thought we were reaching the right people but as often is the case, the people most impacted I don't think got enough say in it..." -Participant 4

\section{Lack of buy-in from medical staff}

The lack of buy-in from frontline healthcare practitioners was identified by all participants as a factor leading to the unsuccessful implementation. Participants believed role clarity and expectations were unclear, and this negatively impacted buy-in. This challenge was augmented by ambiguity around the possible long-term benefits of the program to both patients and providers. The team-based approach to COPD care through smartphone messages was meant to reduce caseload burden in the long-term. However, participants felt this was poorly communicated to providers, and most participants felt the program increased workload.

"I think the primary care docs felt it was an add-on to their regular work. So did their staff. It wasn't clear as to who was going to be responsible for responding to the text messages. It wasn't clear how frequent the text messages would be"-Participant 3

Participants felt this could have been better communicated by first explaining how the CDMI program could complement their day-to-day responsibilities in the long term. To increase buy-in, participants felt that key components should have been better communicated, including implementation instructions, roles and responsibilities, along with the expected benefits. Participants noted a divide between the research team and the healthcare providers on the ground involved in implementation. 
"I think that the folks that were involved with designing the project could see the big picture and could see how this could make things less work in the long run, in exchange for maybe more effort during the study period ... the [medical] teams had voiced concern that they weren't seeing value in proceeding with this [the CDMI project], and as a result they were not very invested in doing this kind of work because to them, it was just extra work." Participant 2

Despite the research team being interdisciplinary, some participants noted an apparent lack of awareness of how the program would interact with daily clinic work, resulting in unrealistic expectations and inadequate planning. One example given was around the lack of consideration for the workload of family physicians, who were tasked with recruitment, which lead to frustration and lack of support. The lack of incentive for participating in CDMI was discussed by participants as contributing to poor overall participation and recruitment.

"So for example the psychiatry and medicine folks I got the feeling that they thought 'well, this should be easy for family docs to identify patients that could be for them easily recruited and to get the cell phones and everything should flow really smoothly', yet the family docs weren't really onboard and recruitment was slow. The staff were not buying into the text messaging thing and I don't think others really appreciated how difficult that was." Participant 3

\section{Inadequate patient engagement}

Three interviewees attributed inadequate patient engagement to implementation failure. They highlighted that patients were not involved in CDMI's planning or design, meaning patient perspectives and preferences were insufficiently incorporated.

“...it probably would have been useful to have a patient involved earlier. We might have learned that they weren't going to use these phones for example..." -Participant 4

Participants discussed challenges they felt patients had encountered such as paying for parking, working with technology, and having to conduct multiple tests. Despite trying to make study appointments around the same time as a patient's regular visit, study appointments were often much longer in duration and required patients to complete extra paperwork. These added inconveniences were a deterrent to overall program momentum. In addition, participants felt technological support for platform's roll-out for both providers and patients was difficult to access, leading to reduced engagement from patients and providers. The technology meant to improve care was perceived as a barrier for patients recruited and also hampered further recruitment efforts.

“... but the patients themselves had difficult using [the smartphone technology] for very practical reasons. Most of the folks with CHF and COPD are not young people, right?... So maybe if we limited the inclusion criteria to a younger batch, maybe the outcome would've been different."” - Participant 2

"...people were sort of already using some of it and then we had some technical glitches too." - Participant 4

\section{Contextual barriers}

Participants acknowledged their context (an academic family health centre) was an important consideration both at the planning level and throughout implementation. An interesting paradox of the interdisciplinary nature of the research team came from the funding requirement for an interdisciplinary team. While touted as being a strength in the planning process, challenges arose with this diverse team during implementation since many of these researchers and practitioners had not regularly worked together prior to CDMI.

“... but I would have to say that the people involved on the [research team] didn't really know each other that well or really had a good understanding of how the other folks on the [research team] conducted their business or at least that was one factor that was kind of missing. I don't know how you correct that really because there was a group from psychiatry, there was a group from internal medicine, and there was family medicine, and then there were other people contributing to the study design and evaluation." - Participant 3

This also brought an additional layer of complexity around evaluation of the programs with different disciplines valuing different metrics.

Lack of program resources posed obstacles to project implementation. This was apparent in the cell phones provided to clinics and patients.

"...the donation phones initially from [provider names] and they were old ... and the buttons are miniscule." - Participant 2

"Yeah, because most people I think especially in the [city] area probably had better [mobile] devices 
already and they weren't wanting to go back [to using older phones]...The telephone company did give us the phones free which was good, but patients didn't really want to use the older phones." - Participant 4

Staff turnover within the CDMI team was discussed as impacting both planning and implementation. During the planning and implementation stages of the project, several key project staff stepped away from their role either temporarily or permanently. Although their roles were filled, this unexpected turnover required additional time to integrate roles, and slowed the momentum.

"I think people were really affected when [Team Member A] was lost to us, although [Team Member B] came in, you know, just it's hard to keep the momentum in the same kind of direction." - Participant 4

The CDMI program required clinic staff to be directly involved in program implementation. Thus, the research team did not have full control over the personnel involved in the project, which was acknowledged to a challenge for implementation.

"I mean we tried to mitigate that [the lack of control over personnel], right, by having the directors of the family health teams be part of the project. But at the end of the day you don't have full control over personnel, and that's reality, right? That's what we learned from this project." -Participant 2

\section{Discussion}

The themes gathered from this study may seem intuitive and they have certainly been demonstrated as important in the literature, however they highlight the complexity and interconnectivity of these factors in practical application. They also demonstrate the need for a strong implementation plan to guide both program planning and implementation processes. Even when individual implementation barriers are accounted for, complex and interconnected barriers may still arise. This is especially true if the proper and continuous engagement of all key stakeholders is not done well. For our study, it was clear that having an interdisciplinary research team was necessary for enhancing the planning process; however, it was not sufficient for identifying all barriers and it may have hindered implementation success.

A poor theoretical basis for implementation guidance can make retrospective analysis of failed implementations difficult [16]. When implementation is guided by theory, it is more likely to succeed. The CDMI program was theoretically grounded during its planning stages
[9], however, the implementation phase lacked theoretical guidance. Even though five of the six theoretical components proposed by Bodenheimer and colleagues [9] were considered in planning (buy-in by health care organizations, self-management support, structured practice teams for chronic care management, decision support and clinical information systems [9]), they lacked practical and contextual consideration for implementation.

With the exception of linkage with community resources, all other elements suggested by Bodenhiemer were incorporated into program planning. First, buy-in was achieved during program planning through an interdisciplinary team, with active participation from family physicians, and specialists in COPD, as well as psychiatry. Second, a central feature of CDMI was a navigator role to enhance patient self-management support and to support program implementation. This self-management support, although well intention, did not work well in practice largely due to insufficient consultation with providers and lack of patient engagement in planning. Third, structured practice teams were enabled through the proposed coordination of the navigator and primary care provider, with guidance from specialists, to share the responsibility of assessing and monitoring patients' through the smartphone platform (including symptoms of depression, anxiety, adherence to medications and follow-up appointments). Fourth, decision support was incorporated through the navigator role who was meant to be available to support implementation and any technology trouble-shooting. Lastly, clinical information systems were a key design feature of the program; existing electronic medical records system were integrated with the programs text messaging to provide reminders to patients and feedback to providers. Linkage with community resources was not considered during program planning or implementation. Our study identified the several shortcomings in the application of the proposed constructs by Bodenheimer and colleagues [9]. First, the lack of patient engagement and provider consultation meant that the program was not aligned with the needs patients or requirements of providers. Second, while buy-in from organizational leads was obtained in planning, similar buy-in from front-line providers for implementation was not achieved. Third, decision support and clinical information systems were felt to duplicate existing services without clarity on expected outcomes for patients or providers. Lastly, interdisciplinary collaboration was, for the most part, only done at the planning level, and less so at the implementation and patient care level.

There are several implementation models that could have been used to support the success of CDMI such as CFIR or the PARiHS (Promoting Action on Research 
Implementation in Health Services) framework. PARiHS defines successful implementation as a function of three factors: evidence, context, and facilitation [17]. Adherence to this framework may have facilitated the necessary 'pre-work', such as conducting a proper needs assessment with patients, staff, and other stakeholders. Ecological theories of implementation such as the Active Implementation Framework [18] or Durlak and DuPre's Ecological Framework [19] support a strong consideration for adaptability to factors like multiple stakeholders, complexities of health care systems, and the interconnectedness of variables [20]. Using a model, theory, or framework to practically support implementation with consideration for context is essential.

The benefits of interdisciplinary collaboration in research and program implementation are noted in the literature [21]. The CDMI research team was interdisciplinary, however, a lack of previous working relationships led to challenges in implementation. While interdisciplinary teams can support the likelihood of creating a successful intervention [22], equally as important is ensuring strong working relationships throughout implementation, building on previous successes (when available), and minimizing the risk to momentum if or when there is team member turnover.

There are several limitations of this study including the small sample size and the specific context in which the research took place. Having more study participants could have helped us develop a more complete picture of why this evidence-based program failed to successfully implement. Our findings are not meant to be generalized to other contexts, instead we believe our rich description of this unsuccessful implementation can provide lessons for other interventions - most notably, the importance of using theory to guide and support both planning and implementation, along with the importance of involving all stakeholders in both of these processes.

\section{Conclusion}

In order to close critical gaps that currently persist in implementation science literature, reporting of implementation efforts including unsuccessful efforts is necessary. Describing unsuccessful interventions will support better understanding of how and why some evidencebased interventions do not succeed within a practical context. Understanding this can also develop our understanding of the complexity and interconnectivity of implementation factors within a specific context. These learnings can support improved methods for differentiating implementation-based failings and interventionbased failings, grow the literature surrounding failed implementation processes, and promote reflective learning from implementation efforts.

\section{Supplementary Information}

The online version contains supplementary material available at https://doi. org/10.1186/s12913-021-06100-4.

\section{Additional file 1.}

Additional file 2.

\section{Abbreviations}

CDMI: Chronic Disease Management Initiative; CFIR: Consolidated Framework for Implementation Research; CHF: Congestive Heart Failure; COPD: Chronic Obstructive Pulmonary Disease

\section{Acknowledgements}

Not applicable.

\section{Authors' contributions}

SLS conceived the research project; SLS supported data collection and led the data analysis. SLS drafted the first version of the manuscript. RM supported data collection and analysis; RM helped with manuscript editing. PC conducted data analysis and supported the development of the manuscript. BL contributed to the drafting and editing of the manuscript. All authors have read and approved the final manuscript.

\section{Author's information}

S.L. Sibbald (BHsc - University of Western Ontario, MSc and PhD - University of Toronto) is currently an assistant professor at Western University within the Faculty of Health Sciences, School of Health Studies. She is also jointly appointed with the Schulich School of Dentistry and Medicine's Department of Family Medicine as well as the Schulich Interfaculty Program in Public Health.

B. Law is an undergraduate student at the University of Western Ontario within the Faculty of Health Sciences.

\section{Funding}

The research in this study was funded by a Dean's Grant from the Schulich School of Dentistry and Medicine, Western University. This publication represents the views of the authors and does not necessarily represent the views of the Schulich School of Dentistry and Medicine or of Western University.

\section{Availability of data and materials}

The datasets analyzed during the study are not publicly available due to the transcripts containing identifiable markers of healthcare providers but are available from the corresponding author on reasonable request after identifying details removed.

\section{Ethics approval and consent to participate}

All participants provided written consent prior to participating. Research ethics approval was provided through Western University's Health Science Research Ethics Board (HSREB) (protocol \#108416).

Consent for publication

Not applicable.

Competing interests

Not applicable.

Received: 18 May 2020 Accepted: 19 January 2021

Published online: 12 February 2021

References

1. Aarons GA, Ehrhart MG, Farahnak LR, Sklar M. Aligning leadership across systems and organizations to develop a strategic climate for evidencebased practice implementation. Annu Rev Public Health. 2014;35(1):255-74.

2. Lau EY, Saunders RP, Pate RR. Factors influencing implementation of a physical activity intervention in residential Children's homes. Prev Sci. 2016; 17(8):1002-11.

3. Stirman SW, Miller CJ, Toder K, Calloway A. Development of a framework and coding system for modifications and adaptations of evidence-based interventions. Implement Sci. 2013;8:65. 
4. Keith RE, Crosson JC, O'Malley AS, Cromp D, Taylor EF. Using the Consolidated Framework for Implementation Research (CFIR) to produce actionable findings: a rapid-cycle evaluation approach to improving implementation. Implement Sci. 2017;12(1):15.

5. Eccles MP, Mittman BS. Welcome to Implementation Science. Implement Sci. 2006;1(1):1. URL at https://implementationscience.biomedcentral.com/ articles/10.1186/1748-5908-1-1.

6. Geerligs L, Rankin NM, Shepherd HL, Butow P. Hospital-based interventions: a systematic review of staff-reported barriers and facilitators to implementation processes. Implement Sci. 2018;13(1):36.

7. Proctor EK, Powell BJ, McMillen JC. Implementation strategies: recommendations for specifying and reporting. Implement Sci. 2013;8:139.

8. Ranji SR, Shojania KG. Implementing patient safety interventions in your hospital: what to try and what to avoid. Med Clin North Am. 2008;92(2): 275-93 vii-viii.

9. Bodenheimer T, Wagner EH, Grumbach K. Improving primary care for patients with chronic illness. JAMA. 2002;288(14):1775-9.

10. Palmier-Claus JE, Rogers A, Ainsworth J, Machin M, Barrowclough C, Laverty $L$, et al. Integrating mobile-phone based assessment for psychosis into people's everyday lives and clinical care: a qualitative study. BMC Psychiatry. 2013;13(1):9

11. Bjerke TN, Kummervold PE, Christiansen EK, Hjortdahl P. "It made me feel connected"-an exploratory study on the use of Mobile SMS in follow-up Care for Substance Abusers. J Addict Nurs. 2008;19(4):195-200.

12. Damschroder $\mathrm{L}$, Aron DC, Keith RE, Kirsh SR, Alexander JA, Lowery JC. Fostering implementation of health services research findings into practice: a consolidated framework for advancing implementation science. Implement Sci. 2009:4:50

13. Hsieh HF, Shannon SE. Three approaches to qualitative content analysis. Qual Health Res. 2005;15(9):1277-88.

14. Clark V, Braun V. Teaching thematic analysis: overcoming challenges and developing strategies for effective learning. Psychologist. 2013;26(2):120-3.

15. Pinnock H, Barwick M, Carpenter CR, Eldridge S, Grandes G, Griffiths CJ, et al. Standards for Reporting Implementation Studies (StaRI): explanation and elaboration document. BMJ Open. 2017:7(4):e013318.

16. Nilsen P. Making sense of implementation theories, models and frameworks. Implement Sci. 2015;10:53.

17. Kitson A, Harvey G, McCormack B. Enabling the implementation of evidence based practice: a conceptual framework. Qual Health Care. 1998;7(3):149-58.

18. Fixsen DL, Naoom SF, Blase KA, et al. Implementation research: a synthesis of the literature: Tampa Florida University of South Florida Louis De La Parte Florida Mental Health Institute National Implementation Research Network; 2005.

19. Durlak JA, Dupre EP. Implementation matters: a review of research on the influence of implementation on program outcomes and the factors affecting implementation. Am J Community Psychol. 2008:41:327-50.

20. McKillop A, Shaw J, Sheridan N, Gray CS, Carswell P, Wodchis WP, et al. Understanding the attributes of implementation frameworks to guide the implementation of a model of community-based integrated health Care for Older Adults with complex chronic conditions: a metanarrative review. Int J Integr Care. 2017;17(2):10

21. Bridle H, Vrieling A, Cardillo M, Araya Y, Hinojosa L. Preparing for an interdisciplinary future: a perspective from early-career researchers. Futures. 2013:53:22-32.

22. Jeffrey P. Smoothing the waters: observations on the process of crossdisciplinary research collaboration. Soc Stud Sci. 2003;33(4):539-62.

\section{Publisher's Note}

Springer Nature remains neutral with regard to jurisdictional claims in published maps and institutional affiliations.

Ready to submit your research? Choose BMC and benefit from:

- fast, convenient online submission

- thorough peer review by experienced researchers in your field

- rapid publication on acceptance

- support for research data, including large and complex data types

- gold Open Access which fosters wider collaboration and increased citations

- maximum visibility for your research: over $100 \mathrm{M}$ website views per year

At BMC, research is always in progress.

Learn more biomedcentral.com/submissions 